\title{
The Toponym Polonia in French Administrative Documents in the 1st Half of the XIVth Century
}

\section{[Le toponyme Pologne dans les documents administratifs français de la 1ère moitié du XIVe siècle]}

\author{
Zdenka Schejbalova
}

\section{DOI: 10.18355/XL.2015.08.02.28-35}

\section{Résumé}

Dans l'article nous étudions le toponyme Pologne qui apparaît en tant que titre du roi titulaire de Pologne, jusqu'en 1335, dans les accords conclus par Jean de Luxembourg, roi de Bohême et comte de Luxembourg, et conservés dans les Archives nationales de Luxembourg et dans les Archives municipales de la ville de Luxembourg. La plupart des accords est rédigée en français, d'autres en latin et allemand. Le toponyme Pologne y est orthographié de maintes façons (22 en français), même s'il y avait plusieurs accords rédigés au même lieu et la même journée. Son orthographe ne s'est pas unifiée tout au long de l'usage. Il en résulte, entre autres, que les accords étaient rédigés par plusieurs scribes ou copistes. Les accords représentent des documents précieux pour la connaissance de l'évolution du français dans sa phase de transformation à la $1^{\text {ere }}$ moitié du XIV ${ }^{\mathrm{e}}$ siècle.

Mots-clés

toponyme Pologne; accords de Jean de Luxembourg; le français de la $1^{\text {ère }}$ moitié du $\mathrm{XIV}^{\mathrm{e}}$ siècle

L'objet de cet article repose sur l'analyse des formes du toponyme Pologne utilisées dans les accords de Jean de Luxembourg conclus à la $1^{\text {ère }}$ moitié du XIV siècle.

Jean de Luxembourg (1296-1346) a épousé la princesse Elisabeth des Přemyslides (1292-1330) à Spire sur le Rhin (Speyer am Rhein en allemand). Le $1^{\text {er }}$ septembre 1310 (Spevacek, 1982: 49), le couple a été couronné au Château de Prague le 7 février 1311 (Spevacek, 1982: 71). Jean de Luxembourg, comte de Luxembourg, reçoit le titre du roi de Bohême et il devient en même temps roi titulaire de Pologne (1310-1335). C'est son titre du roi de Pologne qu'il a hérité de son prédécesseur Venceslas III qui est l'objet de notre analyse linguistique.

Les transcriptions des manuscrits des accords, conservés dans les Archives nationales de Luxembourg et dans les Archives municipales de la ville de Luxembourg, sont classés selon l'ordre chronologique et résumés par Camille Wampach, leur auteur, dans un recueil qui s'intitule Urkunden- und Quellenbuch zur Geschichte des altluxemburgischen Territorien, Urkunden Graf Johanns des Blinden, die Urkunden aus Luxemburger Archivbeständen, Publications du CLUDEM, Luxembourg, 1997. Tout le recueil comporte la transcription de 94 manuscrits au total, dont 20 en latin $(2,3,5,6,7,8,12,13,14,15,18,19,20,21,25,26,48,62,67,77)$, 4 en langues germaniques $(44,49,78,91)$ et 70 en français. Tous sont datés, le premier provient du 5 juillet 1310 et le dernier remonte au 25 mars 1346. La plupart d'entre eux contient aussi l'indication du lieu de l'élaboration de l'accord.

Les accords comprennent des arrangements entre Jean de Luxembourg et d'autres seigneurs, des privilèges concédés aux seigneurs pour des services rendus, aux cloîtres ou aux villes, des quittances et d'autres. Les textes présentent un caractère juridique normatif, disposant d'un style et d'un vocabulaire particulier. 


\section{Origine}

Le toponyme Pologne, en polonais Polska, en tchèque Polsko, en allemand Poland, en latin Polonia vient de la dénomination d'une tribu des Slaves occidentaux des Polanes: Polanie, Poljane (Safarik, 2011: 121-122) (peuple de la plaine - pole - le champ) qui occupait le territoire de la plaine de la rivière Warta au $8^{\mathrm{e}}$ siècle (Ottova encyklopedie obecnych vedomosti, 1997: 35).

Les historiens tels que Małecki Antoni (1907), Dzięcioł Witold (1966), Wandycz Piotr Stefan (1998), Davies Norman (1996), Kosman Marceli (2011), Macůrek Josef (1948) et d'autres traitant de l'histoire de Pologne, y décrivent la généalogie et l'expansion des Polanes.

Dans les plus anciens textes écrits en latin, c'est le chroniqueur allemand Thietmar von Merseburg (975 - 1018) qui mentionne Miesko, duc de Pologne "Unde Miseconis, Poleniorum incliti ducis" (Holtzmann, 1935: 194). Le toponyme Pologne y apparaît sous la forme de Polenia “... a Polenia veniens ..." (Holtzmann, 1935: 518).

Dans la première chronique russe dite Chronique des temps passés ou Chronique de Nestor (По́весть временны́x лет/Несторова летопись) du XII siècle, nous trouvons la distinction des Polanes Poljane dits Lachowie/Ljachowe: “Словъне же ови пришедше и съдоша на Висль, и прозвашася ляховъ, а от тгхх ляховъ прозвашася поляне, ляховъ друзии - лютицг, инии мазовшане, а инии поморяне." (Tvorogova, 2006-2011: 1)

La forme latine de Pulani apparaît dans les Annales Sangallenses Maiores: “1032 ... imperatore per idem tempus Pulanis Sclavis bello insistente.” (Pertz, 1826 : 83)

\section{Analyse du toponyme Pologne dans les accords}

\section{Accords en français}

Dans les accords étudiés du $\mathrm{XIV}^{\mathrm{e}}$ siècle, rédigés en français, le toponyme Pologne apparaît sous plusieurs formes dans le titre du roi de Pologne de Jean de Luxembourg. Nous en avons trouvé 34 occurrences, dont 22 variantes:

- variantes vocaliques dans la syllabe initiale: $\mathrm{Po}-\mathrm{Pou}$-, $\mathrm{Pu}$-: Polane/Poulane/Pulane. - la consonne liquide $l$ simple ou double: Polaine /Pollaine, Poloinel Polloine, apocope Poul/ Poull.

- variantes des monophtongues -la-, -le-, -lo- ou des diphtongues -lai-, -lei-, -loi- et une fois la voyelle nasale -loin- dans la deuxième syllabe: Polane/Poulane/Polene/Polenne/Polone, /Pollaine/Poulainne/Poulleinne/Poloine/Polloine, Pouloingne.

- la consonne nasale $n$ simple ou double dans la troisième syllabe: Polane/Polanne, Polaine/Polainne, Poulaine/Poulainne.

La consonne nasale double renvoie à la nasalisation de la voyelle précédente, à l'époque, au fur et à mesure, dénasalisée dans la syllabe ouverte.

- variantes avec / / / (1 occ.): Pouloingne.

- la syllabe finale - ne $(36$ occ.) ou - nie (1 occ.): Pollonie

Le toponyme apparaît aussi sous la forme abrégée par une apocope: Poull (43, 44), Poul (56), Polle (52), Polon (51).

Le tableau suivant contient, dans la première colonne, le numéro chronologique de l'accord, la deuxième colonne renferme le toponyme, la date de l'accord se trouve dans la $3^{\mathrm{e}}$ colonne et le lieu est indiqué dans la $4^{\mathrm{e}}$ colonne. 


\begin{tabular}{|c|c|c|c|}
\hline 4 & rois de Behaing et de Polane & 25 mai 1314 & \\
\hline 40 & roys de Boeme, de Polanne & 30 août 1334 & Amiens \\
\hline 42 & roys de Boeme, de Poulane & 30 août 1334 & Amiens \\
\hline 54 & roys de Boeme, de Poulane & 20 décembre 1334 & Paris \\
\hline 55 & roys de Boeme, de Poulane & 21 décembre 1334 & Paris \\
\hline 30 & rois de Boeme et de Pulane & 12 janvier 1327 & Bruxelles \\
\hline 53 & roys de Boeme, de Polone & 10 décembre 1334 & Paris \\
\hline 17 & roys de Behaingne et Polenne & $1^{\text {er }}$ juillet 1323 & \\
\hline 34 & rois de Boeme et de Polenne & 31 octobre 1329 & Arlon \\
\hline 57 & roys de Boeme et de Pollene & 21 décembre 1334 & Paris \\
\hline 9 & roys de Behaigne et Poulleinne & 11 juin 1318 & Trier \\
\hline 11 & roys de Beheingne, de Pouloine & 25 juin 1320 & \\
\hline 89 & Bresselau en Pollaine & 4 janvier 1345 & Breslau \\
\hline 29 & roys de Behaigne et de Poulaine & 9 janvier 1327 & Bruxelles \\
\hline 10 & rois de Behaingne et de Poulainne & 16 juin 1320 & Arlon \\
\hline 23 & rois de Boeme, de Poulainne & 25 novembre 1324 & \\
\hline 27 & rois de Boyeme et Poulainne & 27 octobre 1326 & \\
\hline 35 & roy de Boeme, de Poulainne & 3 novembre 1329 & \\
\hline 38 & roys de Boeme, de Poullaine & 29 mars 1333 & Cremona \\
\hline 24 & rois de Behangne et de Polloine & 10 novembre 1324 & \\
\hline 31 & rois de Boeme et de Polloine & 8 avril 1328 & Metz \\
\hline 37 & roy de Boeme et de Poloine & 7 août 1332 & Arlon \\
\hline 28 & roys de Boheme et de Poloine & 8 décembre 1326 & \\
\hline 36 & rois de Boeme et de Pollonie & 3 juillet 1330 & \\
\hline 39 & roy de Boeme et de Pouloingne & 11 avril 1333 & Buxi \\
\hline 22 & roys de Behengne et de Polaine & 23 août 1324 & Trier \\
\hline 41 & roys de Boeme, de Polaine & 30 août 1334 & Amiens \\
\hline 45 & roys de Boeme et de Polaine & 31 août 1334 & Amiens \\
\hline 46 & roys de Boeme et de Polaine & 31 août 1334 & Amiens \\
\hline 43 & roys de Boeme, de Poull & 31 août 1334 & Amiens \\
\hline
\end{tabular}




\begin{tabular}{|c|l|l|l|}
\hline 44 & roys de Boeme, de Poull & 31 août 1334 & Amiens \\
\hline & & & \\
\hline 56 & roys de Boeme, de Poul & 21 décembre 1334 & Paris \\
\hline & & & \\
\hline 51 & rois de Boeme et de Polon & 6 décembre 1334 & Paris \\
\hline & & & \\
\hline 52 & roys de Boeme, de Polle & 6 décembre 1334 & Paris \\
\hline
\end{tabular}

En séjournant à Amiens en août 1334, le roi a fait rédiger 7 accords du $30 \mathrm{au}$ 31 août. Le toponyme Pologne connaît 4 variantes: 3 fois Polaine, 2 fois l'apocope Poull, 1 fois Polanne, 1 fois Pulane.

Le roi poursuit son long voyage à Paris, où il passe le mois de décembre 1334 et quelques mois de 1335 (Spevacek, 1982: 222-223). Nous avons 8 accords à la disposition, dont 6 variantes du toponyme: 2 fois Poulane, 1 fois Polon, 1 fois l'apocope Polle, 1 fois Polone, 1 fois l'apocope Poul, 1 fois Pollene.

Comparé avec les variantes utilisées à Amiens, nous pouvons observer que toutes les formes diffèrent. Apparemment, le roi ne voyageait pas avec sa chancellerie, se déplaçant vite et souvent subitement et à l'improviste (Tadra, 1892: 84). Il profitait, donc, des services de différents scribes locaux. Pourtant, son titre du roi de Bohême reste le même dans tous ces accords. En effet, depuis 1327 jusqu'en 1346 la forme du toponyme Boeme ne change plus.

Dans l'accord rédigé à Paris le $1^{\mathrm{er}}$ janvier 1335 la dénomination du roi de Pologne ne figure plus.

Par la Paix de Trenčín, signé le 25 août 1335, Jean de Luxembourg renonce au titre du roi de Pologne au profit de Casimir III (1333-1370) (Spevacek, 1982: 223).

Dans le diagramme suivant nous pouvons observer le rendement de différentes formes du toponyme Pologne. Les variantes Polaine et Poulainne se montrent les plus productives.

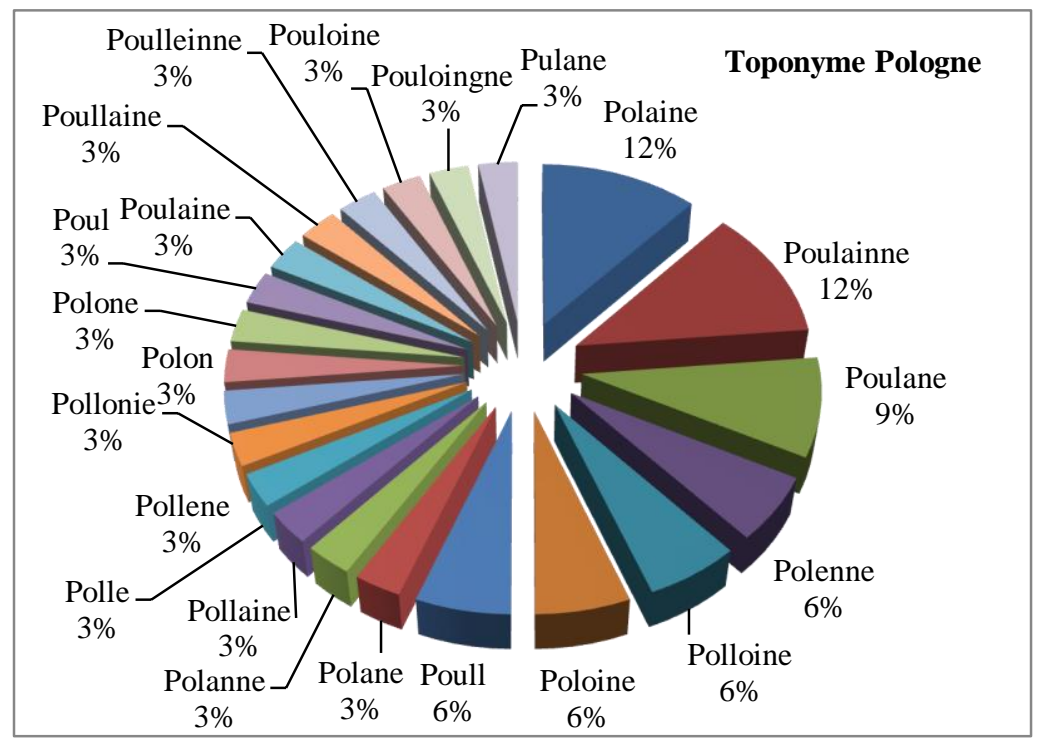




\section{Accords en latin}

Dans les accords rédigés en latin (20 occurrences), 13 d'entre eux contiennent le titre du roi de Pologne (jusqu'en 1334). Il n'y a que deux variantes: 12 fois Polonie rex, depuis le 4 avril 1312 jusqu'en 1334 (3, 5, 6, 7, 8, 12, 13, 14, 15, 18 , 26, 48), 1 fois Pollonie rex (19). Les accords 20, 21, 25 restent sans titre du roi de Pologne.

\begin{tabular}{|c|c|c|c|}
\hline & Nom de lieu & Date & Lieu \\
\hline 2 & $\begin{array}{l}\text { Romanorum regis, } \\
\text { Luczemburgensis et Rupensis } \\
\text { comes necnon marchio Arlunensis }\end{array}$ & 20 septembre 1310 & Colmar \\
\hline 3 & $\begin{array}{l}\text { Bohemie et Polonie rex ac } \\
\text { Lutzelmburgensis comes }\end{array}$ & 4 avril 1312 & Prag \\
\hline 5 & $\begin{array}{l}\text { Boemie et Polonie rex ac } \\
\text { Lucemburgensis comes }\end{array}$ & 12 avril 1315 & Bürglitz \\
\hline 6 & $\begin{array}{l}\text { Boemie et Polonie rex ac } \\
\text { Lucemburgensis comes }\end{array}$ & 31 mai 1316 & Prag \\
\hline 7 & $\begin{array}{l}\text { Boemie et Polonie rex ac } \\
\text { Lucemburgensis comes }\end{array}$ & 30 juin 1317 & Luxembourg \\
\hline 8 & $\begin{array}{l}\text { Boemie et Polonie rex ac } \\
\text { Lucemburgensis comes }\end{array}$ & 13 septembre 1317 & Luxembourg \\
\hline 12 & $\begin{array}{l}\text { de Boemie et Polonie rex } \\
\text { Luccemburgensis comes }\end{array}$ & 12 novembre 1321 & Cambrai \\
\hline 13 & $\begin{array}{l}\text { de Boemie et Polonie rex Luccemb } \\
\text { comes }\end{array}$ & 19 novembre 1321 & Cambrai \\
\hline 14 & $\begin{array}{l}\text { Boemie et Polonie rex } \\
\text { Luccemburgensis comes }\end{array}$ & 7 avril 1322 & \\
\hline 15 & $\begin{array}{l}\text { Bohemie et Polonie rege et comite } \\
\text { Luccemb }\end{array}$ & 17 mai 1322 & Luxembourg \\
\hline 18 & $\begin{array}{l}\text { Boemie ac Polonie rex } \\
\text { Luceburgensis que comes }\end{array}$ & 9 juillet 1323 & $\begin{array}{l}\text { Kloster } \\
\text { Marienthal }\end{array}$ \\
\hline 19 & $\begin{array}{l}\text { Bohemie et Pollonie rex ac } \\
\text { Luccemburgensis comes }\end{array}$ & 13 juillet 1323 & Trier \\
\hline 20 & regis Bohemie & 14 juillet 1323 & \\
\hline 21 & Boemie rege & après 15 juillet 1323 & Luxembourg \\
\hline 25 & $\begin{array}{l}\text { regi Boemie et comitatus } \\
\text { Luccelburgensis }\end{array}$ & 5 mars 1326 & \\
\hline 26 & $\begin{array}{l}\text { Boemie et Polonie rex ac } \\
\text { Luccemburgensis comes }\end{array}$ & 19 septembre 1326 & Luxembourg \\
\hline 48 & $\begin{array}{l}\text { Boemie et Polonie rex ac } \\
\text { Lucemburgensis }\end{array}$ & 19 octobre 1334 & Bruxelles \\
\hline
\end{tabular}

\section{Accords rédigés en allemand}

Il y a 4 accords écrits en allemand dont seulement deux occurrences du toponyme Pologne: Polanen.

\begin{tabular}{|l|l|l|l|}
\hline 47 & $\begin{array}{l}\text { van Byhem, van Polanen ende greve } \\
\text { van Lucelborg }\end{array}$ & 17 octobre 1334 & \\
\hline 49 & $\begin{array}{l}\text { van Byheem, van Polanen ende } \\
\text { grave van Lucelbourgh }\end{array}$ & 31 octobre 1334 & \\
\hline
\end{tabular}




\section{Conclusion}

Le titre du roi de Pologne que Jean de Luxembourg a hérité de Venceslas III, apparait dans 49 accords ( 34 en français, 13 en latin et 2 en allemand) de notre corpus qui compte 94 accords au total. Le premier accord avec le titre est daté du 4 avril 1312 à Prague, le dernier du 21 décembre 1334 à Paris. Dans certains accords avant 1335 le titre n'est même pas mentionné. Or, le corpus étudié ne comporte que les accords qui se sont conservés dans les Archives nationales de Luxembourg et dans les Archives municipales de la ville de Luxembourg. Cependant, nous savons que, définitivement, Jean de Luxembourg n'utilise plus le titre du roi de Pologne après le traité de Trenčín signé le 25 août 1335 .

A la différence du titre du roi de Bohême dont la forme française du toponyme Boeme se fixe depuis 1327, celui de Pologne présente diverses variantes (22 du nombre total de 34 occurrences) jusqu'à la fin de son usage. Une des preuves que le roi (voir les scribes) y accordait moins d'importance en est non seulement que l'orthographe ne s'est pas fixée après un certain temps d'usage, mais aussi que les scribes avaient recourt à l'abrègement par troncation du toponyme (Poul, Poull, Polle).

La richesse de variantes en français renvoie également à la diversité dialectale du français à la première moitié du $\mathrm{XIV}^{\mathrm{e}}$ siècle qui n'obéit, à l'époque, à aucune norme (von Wartburg, 1946, Brunot, 1966, Duchacek, 1963, Ostra, 1980, Sabrsula, 1996). Les scribes dont le nom n'est pas mentionné dans les accords, les ont empreints de leur propre style d'écriture, ainsi que de leur idiolecte.

\section{References}

BRUNOT, F. 1966. Histoire de la langue française. Dès origines à 1900. Tome 1. Paris: Librairie Armand Colin.

CANTEAUT, O. 2002. Une première expérience d'enregistrement des actes royaux sous Philippe le Bel: Le livre rouge de la Chambre des comptes. In: Bibliothèque de l'Ecole des Chartes, vol. 160, issue 1, pp. 53-78+380. ISSN 0373-6237

CHASTANG, P. 2006. Cartulaires, cartularisation et scripturalité médiévale: La structuration d'un nouveau champ de recherche. In: Cahiers de Civilisation Médiévale, vol. 49, issue 1, pp. 21-32. Poitiers: Université de Poitiers. ISSN 0007-9731

CHEVALIER, M. 1977. Un élément du patrimoine géographique Français. Les exonymes. In: Géographie et Cultures, (23), pp. X5-117. Paris: L'Harmattan. ISSN 1165-0354

DAVIES, N. 1996. Boże igrzysko: historia Polski. T. 1.: Od początków do roku 1795. Kraków: Znak. ISBN 83-7006-506-6

DUCHACEK, O. 1963. Historicky vyvoj francouzskeho jazyka. Praha: SPN.

DZIECOE, W. 1966. The origins of Poland. London: Veritas.

HOLTZMANN, R. 1935. Die Chronik des Bischofs Thietmar von Merseburg und ihre korveier Überarbeitung. Berlin: Weidmannsche Buchhandlung.

HOLUB, J. - LYER, S. 1978. Strucny etymologicky slovnik jazyka ceskeho. Praha: SPN.

KINDL, M. - STATNIKOVA, P. 2010. Kralovsky snatek: Eliska Premyslovna a Jan Lucembursky - 1310: pruvodce dobou. Praha: Gallery. ISBN 978-80-86990-67-5

KLADNIK, D. 2009. Prispevek k pomenski razmejitvi terminov endonim in eksonim. In: Acta Geographica Slovenica, vol. 49, issue 2, pp. 393-428, Lubljana: SAZU. ISSN $1581-6613$

KLADNIK, D. 2009. Znacilnosti rabe eksonimov v nekaterih evropskih jezikih. In: Acta Geographica Slovenica, vol. 47, issue 2, pp. 199-222. Lubljana: SAZU. ISSN 1581-6613

KOSMAN, M. 2011. Dejiny Polska. Praha: Karolinum. ISBN 978-80-246-1842-5 
LOICQ, J., 2003. Sur les peuples de nom « Vénète » ou assimilé dans l'Occident Européen. 35, pp. 133-165+428+432. In: Etudes Celtique. Paris: CNRS Editions. ISSN 0373-1928

LUSIGNAN, S. 2003. Chartes et traduction: Les actes Latins et Français de la chancellerie royale et le paradigme de la traduction. In Moyen Français, volume 51-53, pp. 395-420. ISBN 978-2-503-54362-8

LUTTERER, I. - HUŇÁČEK, V. 1976. Původ zeměpisných jmen : etymologický slovník 1000 vlastních jmen zemí, měst a prírodních objektů z celého světa. Praha: Mladá fronta.

MACHAJDÍKOVÁ, B. - TARABA, J. 2012. Urbanitas versus vulgaritas v texte Itinerarium Egeriae. In: Dynamika foriem významu $\mathrm{v}$ románskych jazykoch. Bratislava: Univerzita Komenského Bratislava. ISBN 978-80-223-2553-0

MACUREK, J. 1948. Dejiny polskeho naroda. Praha: Melantrich.

MAŁECKI, A. 1907. Lechici w świetle historycznej krytyki. Lwow: Altenberg.

MOREL, O. 1900. La Grande Chancellerie royale et L'expédition des Lettres Royaux de l'avènement de Philippe de Valois à la fin du XIV siècle (1328-1400). Paris: A. Picard.

ORMELING Sr, F.J. 1980. Exonyms: an obstacle to international communication. In: ITC Journal, vol. 1980-1, pp. 162-176. Enschede: University of Twente, Netherlands. ISSN 0303-2434

OSTRA, R. 1980. Prehled vyvoje romanských jazyku 1. Lidova latina. Francouzstina. Praha: SPN.

OTTOVA encyklopedie obecnych vedomosti na CD ROM, tome XX, Aion CS, 1997. PEHAL, Z. 2012. Toponyma ve smlouvě z roku 1347 na Chotěbořsku - Problém identifikace. In: Acta Onomastica, vol. 53, pp. 219-236. Praha: UJČ AV ČR. ISSN 1211-4413

PERTZ, G. H. 1826. Monumenta Germaniae Historica. Annales Sangallenses Maiores. Tome 1. Hannover. Accessible à <http://www.dmgh.de/de/fs1/object/display/ bsb00000868_00115.html>, (consulté 27/03/2013).

SABRSULA, J. 1996. Vyvoj francouzskeho jazyka. Ostrava: Ostravska univerzita. ISBN 80-7042-455-9

SAFÁRIK, P.J. 1999. Slovanske starozitnosti. Kosice: Oriens. Accessible à <http://zlatyfond.sme.sk/dielo/1522/Safarik_Slovanske-starozitnosti-I>, (consulté 25/ 03/2013), pp. 121-122.

SCHEJBALOVA, Z. 2013. L'exonyme Bohême dans les documents administratifs français de la $1^{\text {ère }}$ moitié du XIV ${ }^{\mathrm{e}}$ siècle. In: XLinguae, vol. 6, issue 2, pp. 2-9. ISSN 1337-8384

SPEVACEK, J. 1982. Kral diplomat. Praha: Panorama.

SRAMEK, R. 1999. Uvod do obecné onomastiky. Brno: Masarykova univerzita. ISBN 80-210-2027-X

STICHAUER, J. 2010. L'évolution de la dérivation suffixale nominale en français préclassique et classique: exemple des déverbaux en - ment. In: Combettes, B., Guillot, C. (Eds.) Le changement en français, vol. 89, pp. 381-402. Bern: Petr Lang. ISBN 978-3-0343-0079-7

TADRA, F. 1892. Kanceláre a pisari v zemich ceských za králu z rodu Lucemburskeho Jana, Karla IV. a Vaclava IV. In: Rozpravy Ceské akademie cisare Frantiska Josefa pro vedy, slovesnost a umení v Praze, année 1, numéro 2. Praha.

TARABA, J. 2005. Du latin à l'ancien français. Bratislava: FF UK.

TARABA, J. 2005. Stara francuzstina z lingvistickeho a prekladatelského hladiska. In: Medzikulturny a medzipriestorovy faktor v preklade. 27. rocník Letnej školy prekladu, pp. 12. ISBN 80-89-137-15-6 
TVOROGOVA, O.V. 2006-2011. Povest' vremennyh let, podgotovka teksta, perevod i kommentarii O. V. Tvorogova Accessible à <http://www.pushkinskijdom.ru/ Default.aspx?tabid=4869>, (consulté 25/03/2013).

WAMPACH C. 1997. Urkunden- und Quellenbuch zur Geschichte des altluxemburgischen Territorien, Urkunden Graf Johanns des Blinden, die Urkunden aus Luxemburger Archivbeständen. Luxembourg: Publications du CLUDEM.

WANDYCZ, P.S. 1998. Stredni Evropa v dejinach od stredoveku do soucasnosti. Praha: Academia. ISBN 80-200-0657-5

WARTBURG, W. 1946. Evolution et structure de la langue française. Bern: A. Francke AG.Verlag.

Words: 2876

Characters: 17891 (9, 94 standard pages)

Dr. Zdeňka Schejbalová, PhD

Department of French Language and Literature

Faculty of Education

Masaryk University

Pořiči 9/11

60300 Brno

Czech Republic

schejbalova@ped.muni.cz 